УДК 330.8

DOI $10.21661 / \mathrm{r}-119552$

\title{
Н.Б. Ефимочкина
}

\section{ОРГАНИЗАЦИОННОЕ ПРОЕКТИРОВАНИЕ: ЗНАЧЕНИЕ, СУЩНОСТНАЯ ХАРАКТЕРИСТИКА}

Аннотация: в статье проанализировано внедрение научных и организационных методов организачионного проектирования систем управления. Автор подробно рассматривает пошаговый алгоритм организаџионного проектирования и показывает реальные механизмы реализации инновационных пилотных проектов и оченки их эффективности.

Ключевые слова: система управления, инновации, введение алгоритма пилотного проекта, декомпозиция работ, структура строителей, блок-схемы, диаграммы Ганта, гистограммы загрузки ресурсов, показатели эффективности, бенчмаркинг.

\section{N.B. Efimochkina}

\section{ORGANIZATIONAL DESIGN: MEANING, ESSENTIAL CHARACTERISTIC}

Abstract: the article analyses the implementation of scientific and organizational methods of organizational design of management system. The author elaborately examines the stepwise algorithm of organizational design and shows the real mechanisms of implementation of innovative pilot projects and assessment of their efficien$c y$.

Keywords: management system, innovations, introduction of pilot project algorithm, work breakdown, builders structure, flowcharts, Gant charts, resource loading histograms, performance indicators, benchmarking.

Научно-организационное проектирование является основой совершенствования систем управления, адаптации хозяйствующих субъектов к современным 
условиям инновационной экономики. Необходимо отметить, что научноорганизационное проектирование как метод повышения эффективности управления, и, в конечном счете, всего производства, ни в коей мере не отрицает и не заменяет традиционные методы - планирование, координирование и т. п. Организационное проектирование необходимо в новых условиях хозяйствования, т.к. традиционные методы в специфических условиях не удовлетворяют потребностям рыночных отношений и, как показывает практика, могут привести к частичной или полной деформации всей системы управления организацией. Исследование методик построения организационных структур, применявшихся до последнего времени, показывает,[1] что они характеризуются нормативным характером и, как правило, сводятся к переносу ранее используемых форм в новые условия. Нередко формирование организационной структуры управления происходит в условиях простого механического копирования структуры вышестоящей организации в нижестоящую, которая отличается только количественно.

Исследователи отмечают априори следующие негативные факторы, применяющиеся при традиционном подходе к организационному проектированию [2]:

1) корректировка численности персонала вместо целей организации;

2) постоянный набор подразделений управления вместо изменения их состава и комбинирования в различных условиях;

3) упор на исполнение неизменных функций в отрыве от постоянно изменяющихся задач;

4) недопустимость изменения организационной схемы, даже при наличии в них недостатков и малой степени пригодности;

5) исследование неформальной структуры организации исключалось из сферы изучения.

Также необходимо учитывать, что, характеризуя научно-организационное проектирование необходимо провести параллели с такой традиционной устоявшейся формой управления как планирование. Планирование определяется 
как процесс выработки и принятия решений, позволяющих обеспечить эффективное функционирование и развитие организации в будущем, в основе которого лежит рациональность распределения ресурсов и функций. Планирование является основой деятельности любого хозяйствующего субъекта и учитывается при формировании организационных структур.

Планирование необходимо, прежде всего, для того, чтобы, имея полную картину имеющихся в конкретный временной отрезок ресурсов, координировать и контролировать механизм их распределения и использования в будущем. Система планирования в организациях реализуется, как правило, на двух уровНях:

1) стратегический уровень планирования - включает оценку будущей жизнеспособности и рентабельности деятельности организации. Реализуется путем разработки комплексного плана развития организации на длительную перспективу (3-5 лет и более). Кроме того, планирование в современных условиях включает комплекс решений и действий по достижению миссии и стратегических целей организации;

2) оперативный уровень планирования - является частью стратегического уровня и детально отражает его содержание, формирует задачи деятельности организации на этапах достижения целей. Реализуется через составление годового плана, в котором по каждому направлению деятельности организации определяются конкретные показатели. Кроме того, годовой план детализируется планами производства и сбыта поквартально и ежемесячно.

Предполагается, что планирование, в том числе и бизнес-планы негосударственных организаций, основывается и впоследствии осуществляется на «запасах»- конкретных ресурсах, имеющихся в распоряжении организации в конкретный временной период, на который составляется план. Но, необходимо учитывать и такое обстоятельство, что служит основой на старте планирования, может перестать являться таковым по мере реализации плана, в случае, если рынок изменит цену товара (услуги), ресурсные характеристики (изменит доступность того или иного ресурса), способ его употребления и т. п. Возникает 
вероятность, что первоначально запланированное действие (количество/качество товара-услуги), не будет реализовано в полной мере, окажется неконкурентоспособным или вообще невостребованным на рынке товаров и услуг. Планирование, по сути, статично (хотя и предусматривает развитие), но не предусматривает выбора поля альтернатив. Кроме того, необходимо отметить, что планирование охватывает, в первую очередь, формальную структуру организации или предприятия и основано на вертикальных связях, характеризующих иерархичность взаимоотношений. Внешне эти связи можно охарактеризовать как механизм «план - отчет». Планирование нормативно, по своей сути, и «человеческий фактор», как показатель неформальной структуры остается за рамками планирования.

Естественно, планирование было и будет в обозримом будущем базовым механизмом в деятельности любого хозяйствующего субъекта, но в современных условиях хозяйствующие субъекты, независимо от их уровня и форм собственности должны находиться в динамичном и непрерывном развитии, которое невозможно обеспечить без исследования тенденций и возможностей, без выбора альтернатив и направлений развития, а в условиях системы управления, основанного на механизмах только жесткого планирования, этого добиться невозможно.

Система управления организациями и предприятиями в рыночных условиях должна обладать высокой гибкостью и мобильностью менять ассортимент, количественные и качественные показатели товаров (услуг), в зависимости от динамично развивающегося спроса на рынке. Это обусловлено тем, что потребности современного рынка делают жизненный цикл продукции (услуг) короче, а разнообразие и ассортимент предлагаемых товаров и услуг диктуют необходимость использовать все имеющиеся ресурсы для повышения конкурентоспособности товара (услуги) конкретной организации. Исходя из этого, современная система управления должна учитывать нестабильность внешней среды, стать адекватной сложным технологиям производства, учитывать возникающие издержки производства и т. п. Конкуренция изменила отношение к качеству 
продукции и уровню обслуживания потребителей, времени выполнения договоров, которые стали очень высокими для традиционных производственных систем, таким образом, рынок требует совершенно новых форм контроля, организации и разделения труда, механизмов принятия управленческих решений, для реализации которых существует объективная потребность и в специальных исследованиях, и в анализе существующего положения организации и выработке новых механизмов управления, отвечающих современным условиям.

Научно-организационное проектирование призвано создать для любой организации дополнительный исполнительный механизм, благодаря которому она будет существовать, мобильно приспосабливаясь к изменяющимся условиям внешней и внутренней среды, и реализовывать свою стратегию. Организационное проектирование направлено на поиск и строительство организационных структур адекватно отвечающих потребностям рынка.

В процесс деятельности по организационному проектированию вырабатывается механизм принятия решений по следующим вопросам (рис. 1). 


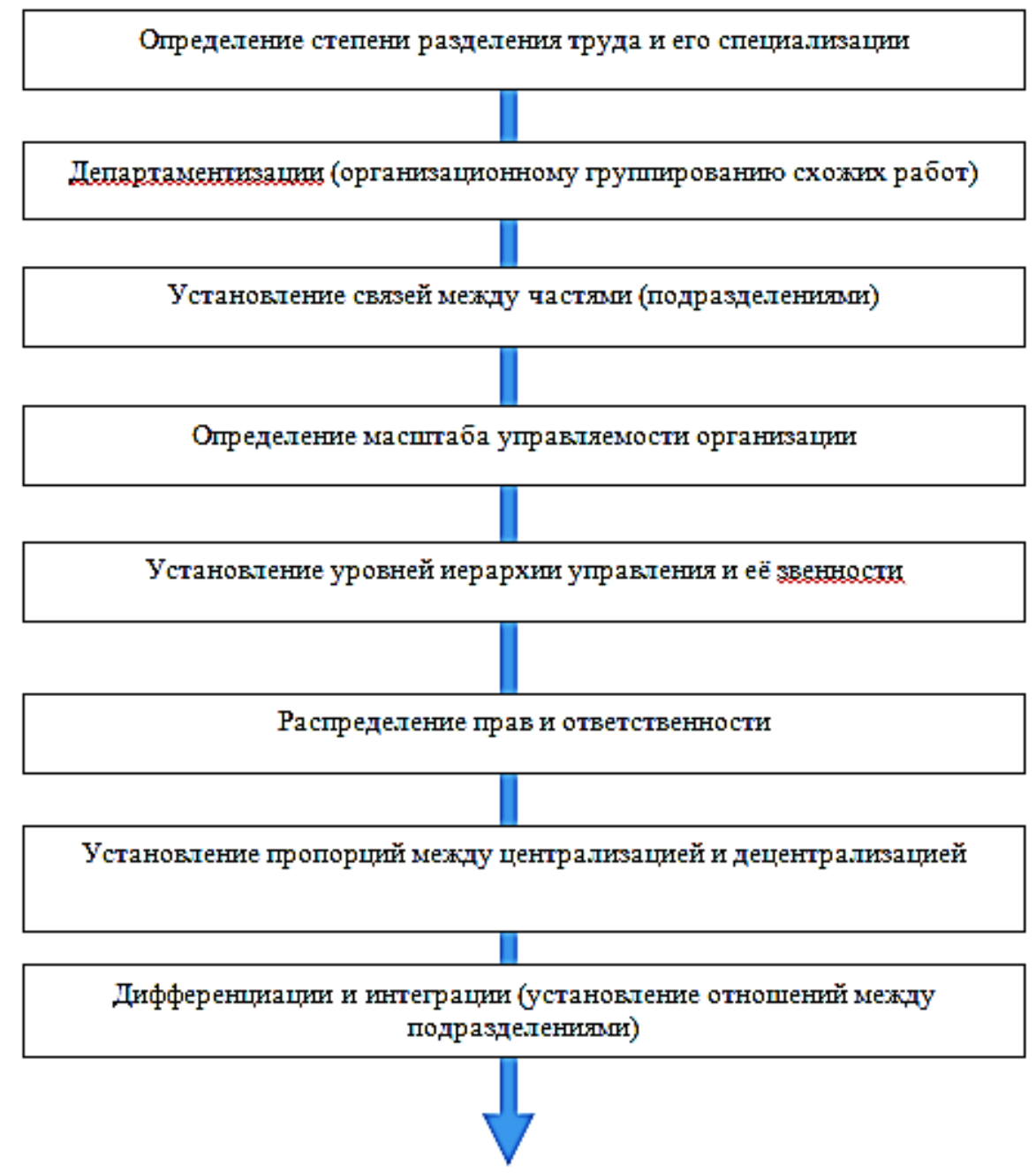

Рис. 1

Из рисунка 1 видно, что в круг вопросов, решаемых в контексте процедуры по научно-организационному проектированию входят механизмы формирования целесообразной управленческой системы, структура которой сможет при минимальных экономических затратах на её содержание мобильно и эффективно обеспечивать достижение стратегических и оперативных целей организации. В результате научно-организационного проектирования компания получает организационную структуру, основанную на рациональном распределении функций (верхнего уровня) по подразделениям, системе делегирования полномочий, системе ответственности и экономичности. Результаты проекта по организационному развитию фиксируются в положениях об организационной структуре, подразделениях и службах. Важно, что результаты оргпроектирования предоставляются не только в виде бумажных документов и схем, но и в виде элек- 
тронных моделей, которые представляют собой комплекс альтернативных стратегий.

Научно-организационное проектирование формирует представление о системе управления и её организационной структуре как о мобильной комплексной системе и позволяет отойти от традиционного понимания организационной структуры системы управления как устоявшейся и неизменной системы органов, каждый из которых соответствует конкретной функции управления.

Научно-организационное проектирование включает в сферу своих интересов такие важные компоненты системы управления как:

- система целей организации и их распределение между подразделениями;

- организационные подсистемы и взаимоотношения между ними,

- распределение задач и функций между подразделениями;

- распределение прав, полномочий и ответственности;

- коммуникации, потоки информации и документооборот (как потоки документированной управленческой информации);

- поведенческие взаимоотношение людей и групп, проявляющиеся при решении общих задач.

Именно такой круг исследований и последующее формирование организационной структуры позволит организации не только мобильно приспособиться к условиям рынка, но и выявить потенциал всех имеющихся ресурсов, сформировать направления стратегии развития. Очевидно, в круг интересов организационного проектирования входят как формальные, так и неформальные механизмы, что делает возможным при проектировании организационных структур управления адаптировать традиционные механизмы управления, научные методы и принципы формирования управленческих структур, такие как системный подход, организационное моделирование, экспертно-аналитическое исследование.

Исходя из вышеизложенного, можно констатировать, что научноорганизационное проектирование - это вид деятельности, направленный на 
развитие и совершенствование системы управления в соответствии с постоянно изменяющимися внешними и внутренними условиями.

Объектом научно-организационного проектирования является система управления конкретной организации или её отдельный сектор (направление деятельности).

В процессе научно-организационного проектирования выявляются такие характеристики организации как объекта исследования:

1) роль и место данной организации в отрасли;

2) состояние производственно-хозяйственной деятельности организации;

3) производственная структура организации;

4) система управления и ее организационная структура;

5) особенности взаимодействия предприятия с внешними организациями;

6) инновационные потребности (возможные связи данной организации с научно-исследовательскими и проектно-конструкторскими организациями);

7) формы и методы стимулирования и оплаты труда сотрудников.

Проведение научно-организационного проектирования может осуществляться в следующих случаях:

- при совершенствовании системы управления действующей организации;

- при разработке системы управления вновь создающейся организации;

- при совершенствовании системы управления производственных объединений или предприятий в период реконструкции или технического перевооружения;

- при совершенствовании системы управлении вследствие изменения формы собственности.

Говоря о целях научно-организационного проектирования, необходимо отметить, что данная процедура часто носит инициативный характер, следовательно, организационное проектирование зависит от потребностей и возможностей организации. Исходя из этого, можно утверждать, что целеполагание организационного проектирования универсально. Тем не менее, основной цуелью ор- 
ганизационного проектирования и комплексного исследования системы управления, её организационной структуры является поиск путей и методов совершенствования системных характеристик.

К таким характеристикам с позиции общего менеджмента относятся:

- цели системы управления;

- функции управления;

- управленческие решения;

- структура управления,

Конечной цุелью научно-организационного проектирования является разработка и внедрение выбранной эталонной модели системы управления.

Задачами научно-организационного проектирования являются:

- постановка целей и задач системы управления организации;

- определение состава и места подразделений управления;

- их ресурсное обеспечение (включая численность и потенциал работающих);

- разработка регламентирующих процедур и документов, закрепляющих и регулирующих формы, методы и процессы, которые осуществляются в организационной системе управления.

В результате проведения процедуры научно-организационного проектирования формулируются конкретные предложения по совершенствованию системы управления организацией, реализация которых позволит:

1) достигнуть оптимального соотношения между управляемой и управляющей подсистемами (сюда входят показатели норм управляемости, показатели эффективности работы аппарата управления, сокращение затрат на управление);

2) повысить производительность труда управленческих работников и рабочих производственных подразделений;

3) улучшить использование материальных, трудовых, финансовых ресурсов в управляющей и управляемой подсистемах;

4) снизить затраты на продукцию или услуги и повысить их качество. 
Для решения задач и достижения целей научно-организационного проектирования, независимо от того, исследуется ли вся система управления организацией, или же управления конкретных секторов (направлений деятельности или филиалов и т. п.), должно быть положено исследование таких факторов организованности системы управления, как:

1) детерминированность всех элементов системы управления. Данный фактор отражает организацию взаимодействия подразделений органов управления. Сущность исследования детерминированности данных элементов заключается в выявлении степени влияния деятельности одного подразделения на другие. Отсутствие такого влияния со стороны конкретного подразделения говорит о его неспособности реализовать одну из целей функционирования организации и, следовательно, делает его лишним в данной системе управления;

2) способность при внешних и/или внутренних потрясениях оставаться некоторое время в неизменном качественном состоянии. Исследование данного фактора позволит определить устойчивость данной системы управления к динамике изменений внешней и внутренней среды;

3) наличие в системе функционирующего управляющего элемента. Под управляющим элементом подразумевается руководитель конкретного уровня. Исследование данного фактора включает в себя изучение сферы компетенции конкретного руководителя, условий его работы, позволяющие/или нет выполнять возложенные на него управленческие задачи и т. п. Выявление участков, на которых отсутствует такой управляющий элемент или же отсутствие у руководителя конкретного уровня, например, определенных компетенций приводит к принятию субъективных управленческих решений и, так называемому, волевому стилю руководства, с соответствующими последствиями;

4) наличие контролирующего элемента. Под контролирующим подразумевается наличие элемента, осуществляющего контроль состояния субъекта управления и оказывающего управляющее воздействие как на сам субъект, так и на любой элемент системы управления; 
5) наличие в системе управления каналов прямой и/или обратной связи. Исследование данного фактора позволит выявить наличие или отсутствие подобных связей, сформировать механизмы регламентации деятельности по приему и передаче информации при подготовке управленческих решений;

6) наличие устойчивых поведенческих групп. Исследование данного фактора подразумевает анализ неформальной структуры организации или исследуемого подразделения, выявление негативных тенденций, потенциала коллектива.

Эффективность исследования и анализ факторов, характеризующих организационную структуру управления, не может быть оценена, исходя только из одного показателя. Таким образом доказано, что исследование и анализ системы управления, её организационной структуры, требуют комплексного структурного подхода, при котором учитываются все показатели, их характеризующие, среди которых - методы управления, технология управления, организационная структура, кадры управления, технические средства управления, информация.

Системный подход позволяет исследовать и связи объекта между элементами, и внешние связи объекта, позволяющие рассматривать его как подсистему для более высокого уровня, и функции управления обеспечивающие принятие управленческих решений заданного уровня качества при минимальных затратах на управление или производство, и механизмы оценки результатов управленческой деятельности и затрат на содержание аппарата управления и т. п.

Важным и основополагающим критерием в методологии научноорганизационного проектирования конкретной организации является четкое формулирование стратегии самой социально-экономической системы - организации.

Определение стратегии организации является первостепенной задачей, независимо от целей, которые определены для научно-организационного проектирования (исследуется вся система управления, или её сектор). Исходное определение глобальной цели организации и её функционирования, которые обуславливают структуру задач и содержание функций аппарата управления 
(классификация целей организации), в конечном итоге, определяют систему уровней управления, эффективность её организационной структуры. Пример указанной классификации организации показан на рис. 2.1.2.

Причем, выявление и формулировка всей совокупности целей, их классификация позволяет выявить четкую структуру целей функционирования на каждом уровне системы управления. Подобная структуризация показывает, насколько они отвечают глобальным интересам. В таком случае дерево целей организации будет наиболее полным. На рис. 2.1.3. представлен фрагмент структуризации целей организации.

Структуризация целей осуществляется для формализованного отображения процесса их распределения по уровням управления. Посредством структуризации описывается их состав, взаимосвязь, упорядоченная иерархия. Это является залогом успешной реализации функциональной стратегии организации.

При этом необходимо учитывать, что многообразие целей на высшем, среднем или низшем уровнях управления не может быть приведено к одному знаменателю. Если глобальную цель организации, в целом, можно охарактеризовать (с позиций общественных интересов) как удовлетворение рыночных потребностей в производимой продукции и услугах, то функциональные цели характеризуют только одну из необходимых для данной организации сторон её функционирования. Вместе с тем, в соответствии с определением Б.3. Мильнера, не может быть и однозначным соответствие между системой целей и организационной структурой управления. На рис. 2. показана общая последовательность перехода от целей организации к её структуре.

Как видно из рис. 4 эффективность организационной структуры управления не может быть оценена только одним показателем. Исследование организационной структуры управления необходимо рассматривать в единой системе: все факторы, характеризующие и влияющие на деятельность системы управления. Эти факторы имеют различную природу, в зависимости от которой формируются специфические методы организационного проектирования, каждый из которых должен применяться в органическом сочетании с другими. 
Важным условием организационного проектирования, характеризующим его эффективность, является не только линейное выявление критериев и характеристик, определяющих сущность факторов, влияющих на организационную структуру управления, но и формирование процедуры прикладного применения всех показателей исследования. Необходимо сформировать такие гибкие критерии и показатели, которые органично впишутся в существующую систему управления, реорганизует её с наименьшими ресурсными потерями.

Для этого в круг вопросов научно-организационного проектирования должны непременно входить следующие характеристики:

1) выявление общих тенденций развития данной организации, её места и роли в современной рыночной экономике;

2) установление особенностей функционирования организации и ее отдельных подразделений;

3) выявление условий, обеспечивающих достижение поставленных целей;

4) определение условий, препятствующих достижению целей;

5) сбор необходимых данных для проведения анализа и разработки мероприятий по совершенствованию действующей системы управления;

6) использования передового опыта других организаций и предприятий;

7) изучение необходимых сведений для адаптации выбранной (синтезированной) эталонной модели к условиям рассматриваемого предприятия. 


\section{По периоду установления: \\ - стратегические (долгосродные, среднесродные, краткосродные);}

- тактиреские

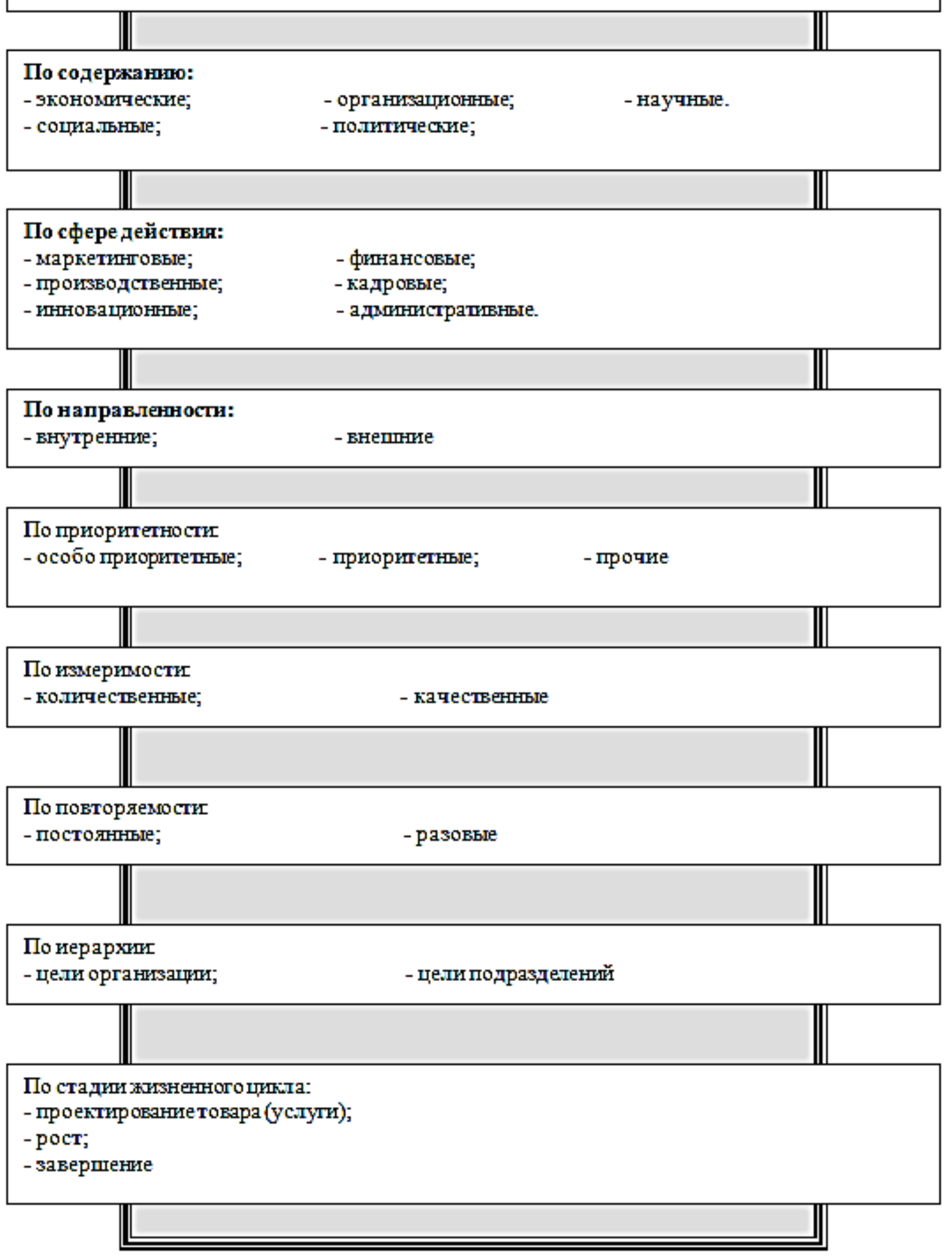

Рис. 2. Классификация целей организации 


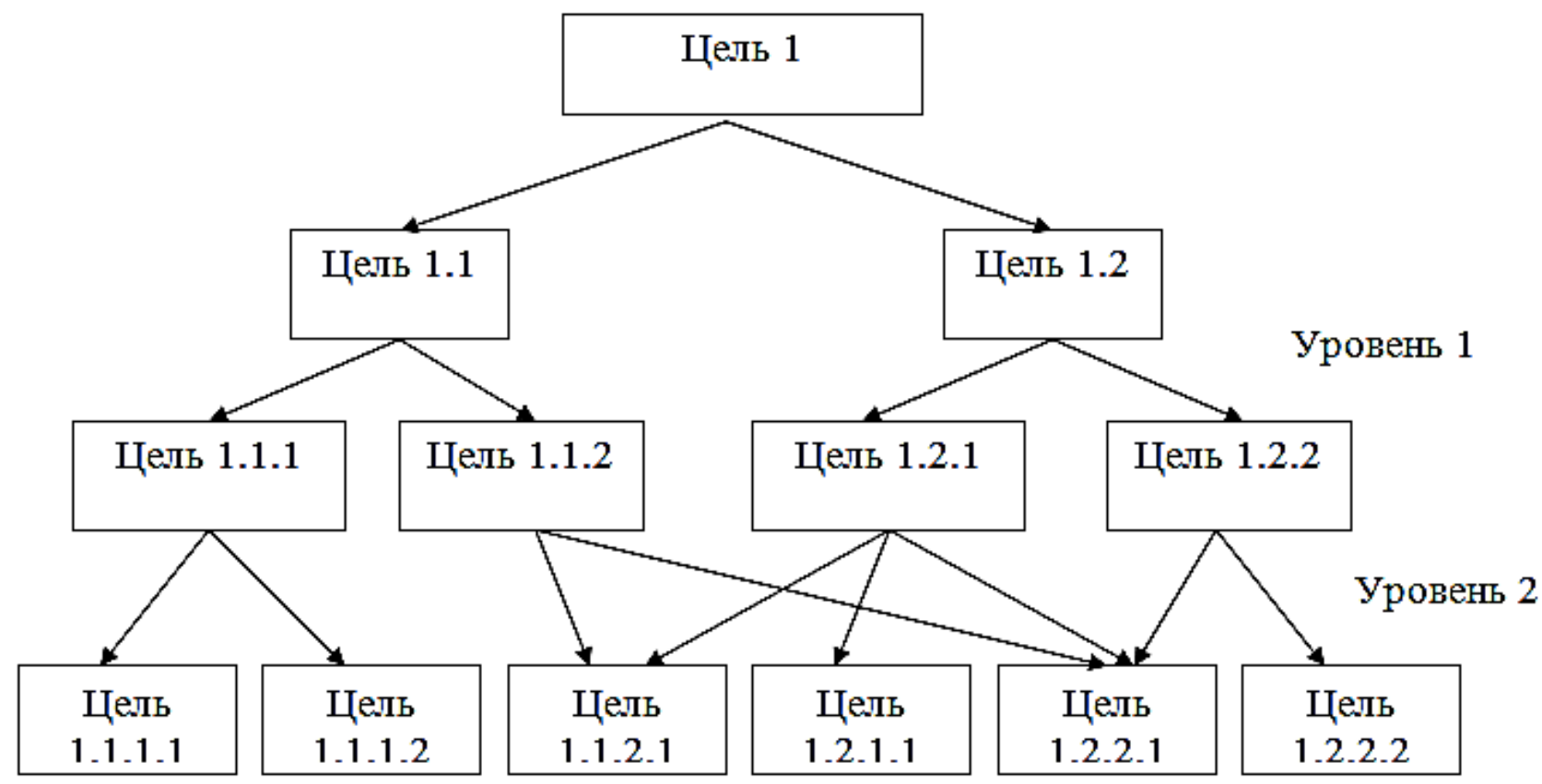

Рис. 3. Фрагмент структуризации целей организации по уровням управления (фрагмент древа целей)

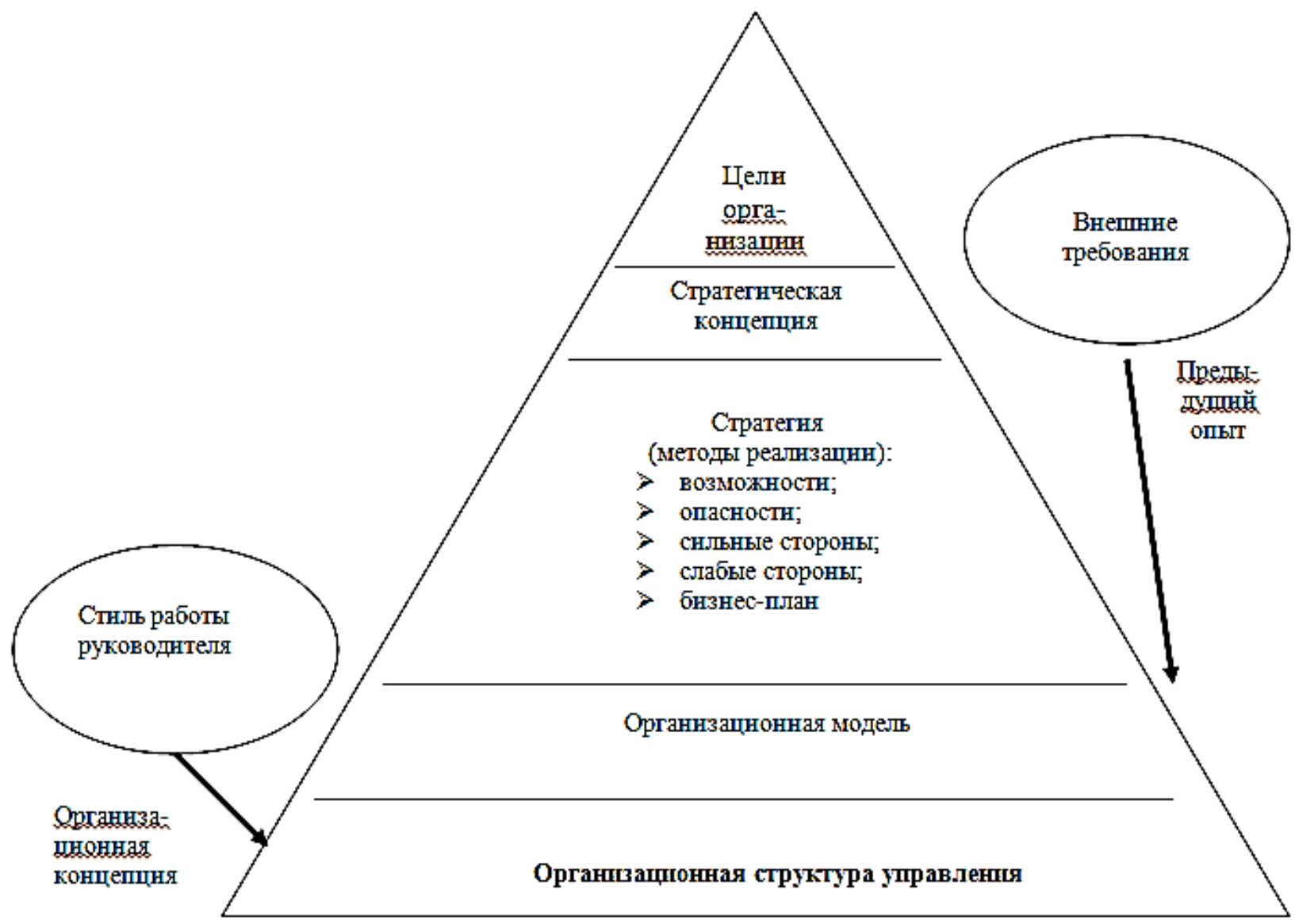

Рис. 4. Переход от целей организации к её структуре

Характеризуя проблематику научно-организационного проектирования необходимо отметить, что оно является научно-прикладным механизмом со- 
вершенствования системы управления в рамках менеджмента организации. В условиях рыночной экономики организационное проектирование становится базой для организационных нововведений, основанной на совокупности методов организационного и технико-экономического исследования всех указанных выше факторов и системных характеристик конкретной организации.

\section{Сиисок литературы}

1. Мильнер Б.3. Теория организации. - М.: Инфра-М, 2016. - с. 146.

2. Мильнер Б.3. Теория организации. - М.: Инфра-М, 2016. - с 159.

Ефимочкина Наталья Борисовна - канд. социол. наук, доцент кафедры «Экономическая теория» ФГБОУ ВО «Российский государственный университет нефти и газа им. И.М. Губкина», Россия, Москва.

Efimochkina Natalia Borisovna - candidate of sociological sciences, associate professor of "Economic Theory" Department FSBEI of HE "I.M. Gubkin Russian State University of Oil and Gas", Russia, Moscow. 